\title{
Kondo effect and spin filtering in triangular artificial atoms
}

\author{
Gergely Zaránd,* a b Arne Brataas, ${ }^{\text {a c }}$ and David Goldhaber-Gordon ${ }^{\mathrm{d}}$ \\ ${ }^{a}$ Lyman Laboratory of Physics, Harvard University, Cambridge MA 02145, USA \\ ${ }^{b}$ Department of Theoretical Physics, Institute of Physics, Budapest University of Technology and \\ Economics, H-1521 Hungary \\ ${ }^{c}$ Department of Physics, Norwegian University of Science and Technology, N-7491 Trondheim, Norway \\ ${ }^{\mathrm{d}}$ Department of Physics and Geballe Laboratory for Advanced Materials, Stanford University, Stanford \\ CA 94305
}

We study strongly correlated states in triangular artificial atoms. Symmetry-driven orbital degeneracy of the single particle states can give rise to an $S U(4)$ Kondo state with entangled orbital and spin degrees of freedom, and a characteristic phase shift $\delta=\pi / 4$. Upon application of a Zeeman field, a purely orbital Kondo state is formed with somewhat smaller Kondo temperature and a fully polarized current through the device. The Kondo temperatures are in the measurable range. The triangular atom also provides a tool to systematically study the singlet-triplet transitions observed in recent experiments $[4,11]$.

Introduction. - Submicron boxes of electrons known as "quantum dots" or "artificial atoms" have proved a fruitful playground for studying single-particle quantized states, interaction, and spin $[1,2]$. The role of orbital degeneracy in the formation of strongly correlated states in artificial atoms has not received the scrutiny it deserves, perhaps because orbital degeneracy is so hard to control experimentally in these systems. Accidental degeneracies may result in spin $S>1 / 2$ states with interesting physical properties [3], and in artificial atoms with almost degenerate states a singlet-triplet transition may also occur, giving rise to interesting non-monotonic behavior in the temperature dependence of the conductance [35]. However, a typical artificial atom, unlike its namesakes, has no spatial symmetries and hence no orbital degeneracies. Instead, its ladder of orbital states is well-described by random matrix theory $[6,7]$ This lack of symmetry seems intrinsic to structures studied by lateral transport through a patterned 2-dimensional electron gas (2DEG), as electrons must enter and exit from leads which break the symmetry of the confinement potential. In contrast, structures studied by vertical transport may have cylindrical symmetry — such ar- tificial atoms exhibit beautiful shell structure [8], demonstrating that geometry can control a level spectrum. Combining the advantages of vertical structures (controlled level spectrum) and lateral structures (controlled tunneling strength) would be very desirable. Here we show that this goal can be achieved by carefully choosing the shape of a lateral structure.

In a perfectly symmetrical triangular artificial atom (TA) each single particle state can be labeled by its corresponding symmetry representation (see Fig. 1). Generically about half the levels are orbitally degenerate. To see this, let us imagine slightly distorting a perfectly cylindrical box to a triangular shape. Representation theory implies that states with angular momentum $L_{z}= \pm 3 n$ split into one-dimensional $\Gamma_{1}$ and $\Gamma_{2}$ representations, while states with $L_{z}= \pm(3 n+1)$ or $L_{z}= \pm(3 n-1)$ remain degenerate, forming two-dimensional $\Gamma_{3}$ representations. As a result, from $N$ originally degenerate states about $2 N / 3$ remain twofold degenerate, while the remaining $\sim N / 3$ levels split into $\sim 2 \times N / 3$ non-degenerate levels by the triangular symmetry.

As we discuss below, a singly-occupied $\Gamma_{3}$ state gives rise to an $S U(N)$ Kondo state with $N=4$, 


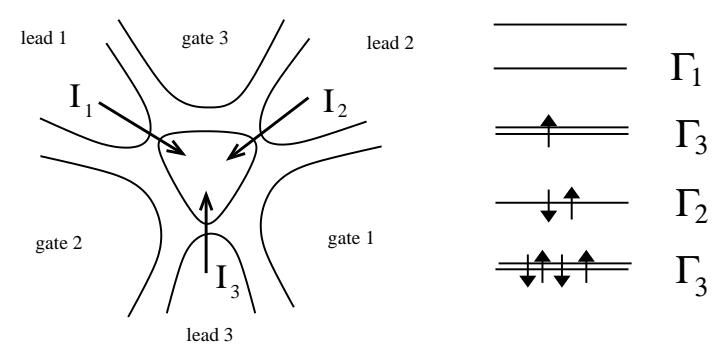

Figure 1. Proposed arrangement with triangular symmetry and the structure of the four-fold degenerate ground state of the isolated TD.

reflecting the fourfold degeneracy of the ground state of the isolated TA (see Fig. 1). In this state orbital and spin fluctuations are entirely entangled. As opposed to the usual Kondo state with phase shift $\pi / 2$, this $S U(4)$ Kondo state is characterized by a phase shift $\delta=\pi / 4$, and is very similar to states proposed recently for artificial molecules (two coupled artificial atoms) [9]. The unusual phase shift could be detected by integrating the TA into an Aharanov-Bohm geometry [10], but could also be inferred from more standard transport measurents. Application of a Zeeman field removes the spin degeneracy of the ground state and drives the system to a purely orbital Kondo state, in which the triangular atom acts as a spin filter, transmitting only electrons with spin aligned to the external field.

The major difference between the artificial molecule system of Ref. [9] and the present triangular arrangement is that in the former case the orbital states of the full system are never truly degenerate because of tunneling between the two sites, while in a perfectly symmetrical TA the $\Gamma_{3}$ states are far closer to degeneracy, split only by a small spin-orbit interaction. Furthermore, the $\Gamma_{3}$ electronic wave functions on the TA strongly overlap with each other, producing a large Hund's rule coupling, and hence a triplet ground state for double occupancy of the four-fold degenerate state. Distorting the shape of the TA with an external gate would gradually split the degenerate $\Gamma_{3}$ states. Therefore, in this geom- etry one could systematically study the singlettriplet transition without applying any external magnetic field $[4,11]$.

Model. - First we focus on the charging of a $\Gamma_{3}$ multiplet. At the Hartree-Fock level the isolated TA can be described by:

$$
\begin{aligned}
H_{\mathrm{TA}}= & \sum_{\tau, \tau^{\prime}, \sigma} d_{\tau \sigma}^{\dagger}\left(E_{\tau \tau^{\prime}}+\Delta E \delta_{\tau \tau^{\prime}}\right) d_{\tau^{\prime} \sigma}-J \vec{S}^{2} \\
& +\frac{E_{C}}{2}\left(n_{+}+n_{-}\right)^{2}+\frac{\tilde{E}_{C}}{2}\left(n_{+}-n_{-}\right)^{2},
\end{aligned}
$$

where $d_{\tau \sigma}^{\dagger}$ creates an electron on the TA within the $\Gamma_{3}$ multiplet with spin $\sigma$ and orbital label $\tau=$ \pm . The energy shift $\Delta E$ is proportional to the (symmetrically-applied) gate voltage and controls the charge on the atom, while $E_{\tau \tau^{\prime}}$ accounts for the splitting generated by deviations from perfect triangular symmetry $\left(\sum_{\tau} E_{\tau \tau}=0\right)$. We denote the total number of electrons in state $\tau= \pm$ by $n_{\tau} \equiv \sum_{\sigma} d_{\tau \sigma}^{\dagger} d_{\tau \sigma}$, and $\vec{S}=\frac{1}{2} \sum_{\tau, \sigma, \sigma^{\prime}} d_{\tau \sigma}^{\dagger} \vec{\sigma}_{\sigma \sigma^{\prime}} d_{\tau \sigma^{\prime}}$ is the total spin of the atom. The terms proportional to $E_{C}$ and $\tilde{E}_{C}$ are generated by the Hartree interaction, while that proportional to $J$ in Eq. 1 is the Hund's rule coupling, generated by exchange.

We describe the attached leads by

$H_{\text {leads }}=\sum_{\varepsilon, \sigma} \sum_{j=1}^{3} \epsilon a_{\varepsilon \sigma j}^{\dagger} a_{\varepsilon \sigma j}$

where $a_{\varepsilon \sigma j}^{\dagger}$ creates an electron in lead $j$ with energy $\varepsilon$ and $\operatorname{spin} \sigma$, and $\left\{a_{\varepsilon \sigma j}^{\dagger}, a_{\varepsilon^{\prime} \sigma^{\prime} j^{\prime}}\right\}=$ $\delta_{j j^{\prime}} \delta_{\sigma \sigma^{\prime}} \delta\left(\varepsilon-\varepsilon^{\prime}\right)$.

For a symmetrical TA we may usefully introduce a new conduction electron basis through the unitary transformation, $a_{m} \equiv \sum U_{m j} a_{j}$, with $U_{m j}=e^{i 2 \pi m j / 3} / \sqrt{3}$. States with $\tau \equiv m= \pm 1$ transform as $\Gamma_{3}$, while $a_{m=0}$ transforms as $\Gamma_{1}$, and cannot hybridize with the $\Gamma_{3}$ doublet on the atom. Therefore, in this new basis, for a perfectly symmetrical TA the hybridization between the atom and the leads takes on a particularly simple form:

$H_{\mathrm{hyb}}=V \sum_{\tau, \sigma}\left(d_{\tau \sigma}^{\dagger} \psi_{\tau \sigma}+\right.$ h.c. $)$,

with $\psi_{\tau \sigma} \equiv \int d \varepsilon a_{\varepsilon \sigma \tau}$. 


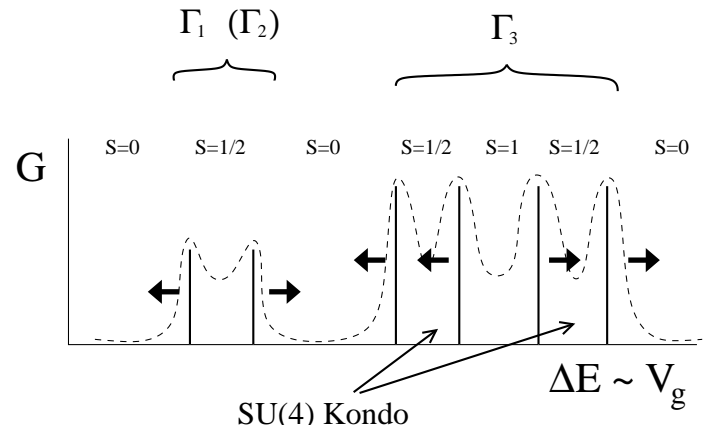

Figure 2. Structure of Coulomb blockade peaks as a function of gate voltage, $V_{g}$. The total number of electrons on the dot increases by one at each Coulomb blockade peak with increasing $V_{g}$. The dashed line indicates the contribution from coherent (Kondo) processes for a TA with spin $S \neq 0$. Application of a Zeeman field shifts the peaks in directions indicated by the arrows.

Coulomb blockade peaks. - We are most interested in the case of a four-fold degenerate ground state arising from a singly-occupied $\Gamma_{3}$ level. ${ }^{1}$ Such a state can be identified experimentally by looking at the height, magnetic field, and temperature dependence of the Coulomb blockade peaks. In the regime where the temperature $T$ is smaller than the level spacing $\Delta$ of the dot, but is still larger than any Kondo temperature or the tunneling rate $\sim 2 \pi V^{2}$ to the leads, the conductance around the Coulomb blockade peaks will be dominated by sequential tunneling. We use a standard rate equation formalism [12] to compute the linear conductance of the TD in this regime. For a symmetrical system the currents $I_{j}$ between leads $j$ and the atom are related to the voltages $V_{j}$ applied on them by the conductance tensor, $I_{j}=\sum_{j^{\prime}} G_{j j^{\prime}} V_{j^{\prime}}$, where $G_{j j^{\prime}}=\frac{3}{2} G \delta_{j j^{\prime}}-G / 2$. A schematic plot of the conductance $G$ is shown in Fig. 2. The four peaks associated with the $\Gamma_{3}$ state are spaced regularly in gate voltage, and their height turns out to be numerically almost

\footnotetext{
${ }^{1}$ Similar considerations would apply for the case of a single hole on the $\Gamma_{3}$ multiplet.
}

identical: For the first and last peak we find $G_{1, \max }^{\Gamma^{3}}=G_{4, \max }^{\Gamma^{3}}=0.9309 \frac{2 e^{2}}{h} \frac{\Gamma}{T}$, while the second and third have a height $G_{2, \max }^{\Gamma^{3}}=G_{3, \max }^{\Gamma^{3}}=$ $0.9019 \frac{2 e^{2}}{h} \frac{\Gamma}{T}$, with $\Gamma=2 \pi V^{2} / 3 \hbar$ the single particle tunneling rate between the atom and one of the leads. Applying a Zeeman field to the atom (i.e., a field parallel to the underlying 2dimensional electron gas), shifts the positions of the peaks in the directions indicated by the arrows in Fig. 2 and also somewhat decreases their height. The $\Gamma_{3}$ peaks are further distinguished by the temperature dependence of the conductance between them, since at low temperatures this valley conductance increases for degenerate ground states due to coherent Kondo-type correlations (see Fig. 2) [13].

The SU(4) Kondo state. - From now on we shall focus on the four-fold degenerate ground state with $n_{+}+n_{-}=1$, expanded in states $|\mu\rangle \equiv|\tau \sigma\rangle$. When coupled to leads, this state exhibits a novel and interesting Kondo effect. We compute the effective interaction between the leads and the atom by integrating out virtual fluctuations to the $n_{+}+n_{-}=0$ and $n_{+}+n_{-}=2$ states, giving:

$H_{\mathrm{int}}=\sum J_{\alpha \beta}^{\mu \nu}|\mu\rangle\langle\nu| \psi_{\alpha}^{\dagger} \psi_{\beta}$,

where $\mu, \nu, \alpha$, and $\beta$ stand for the possible combinations of orbital and spin indices, $\alpha, \ldots, \nu \in$ $\{\sigma, \tau\}$. The couplings $J_{\alpha \beta}^{\mu \nu}$ are typically of or$\operatorname{der} V^{2} / E_{C}$ and their explicit expression is rather complicated. Fortunately, this 'bare' Hamiltonian simplifies considerably upon scaling - a renormalization group analysis reveals that at small energy scales (temperatures) the TA is simply described by the effective exchange Hamiltonian [14]:

$H_{\mathrm{eff}}(T \rightarrow 0)=\tilde{J} \sum_{\alpha, \beta=1, . ., 4}|\beta\rangle\langle\alpha| \psi_{\alpha}^{\dagger} \psi_{\beta}$.

For a TA with perfect symmetry, this Hamiltonian produces a strongly correlated Kondo state with $\mathrm{SU}(4)$ symmetry and entangled spin and orbital degrees of freedom. At $T=0$ temperature, both orbital and spin indices are conserved and the transmitted electrons acquire only a phase shift $\delta_{m, \sigma}$. In this Kondo state all four phase 
shifts equal in zero external field, and following the Friedel sum rule satisfy $\delta_{m, \sigma}=\pi / 4$ [15]. This unusual phase shift should be measurable experimentally in Aharanov-Bohm arrangements [10]. In case of a finite Zeeman field the phase shifts acqire a spin dependence, but they remain independent of the orbital indices, $\delta_{m= \pm 1, \sigma}=\delta_{\sigma}$, though $\delta_{\sigma}$ are related through the Friedel sum rule as $\delta_{\uparrow}+\delta_{\downarrow}=\pi / 2$.

Knowledge of the phase shifts allows us to compute the linear DC conductance through the TA at $T=0$ using the Landauer-Buttiker formalism $[3,16]$. In the ground state, the $m=0$ combination of the leads is totally decoupled, and therefore $\delta_{0}=0$. We can thus construct the scattering matrix $S_{m m^{\prime}}^{\sigma}$ within the basis $\left\{a_{m \sigma}\right\}$, and then express the scattering matrix $S_{i j}^{\sigma}$ by just rotating back to the original basis: $S_{i j}^{\sigma}=$ $\sum_{m, m^{\prime}} U_{i m}^{\dagger} S_{m m^{\prime}}^{\sigma} U_{m^{\prime} j}$. The conductance matrix can then be expressed through $S_{i j}^{\sigma}$, and for a perfectly symmetrical geometry we find that $G(T \rightarrow$ $0)=G_{Q} \frac{4}{9} \sum \sin ^{2} \delta_{\sigma}$ where $G_{Q}=2 e^{2} / h$ is the quantum conductance.

For general $J_{\alpha \beta}^{\mu \nu}$ the Kondo temperature $T_{K}$ cannot be given in a closed form. If, however, the dominant charge fluctuations are to the $n_{+}+$ $n_{-}=0$ state, then $J_{\alpha \beta}^{\mu \nu} \approx J \delta_{\beta}^{\mu} \delta_{\alpha}^{\nu}$, and in the leading logarithmic approximation $T_{K} \approx \Delta e^{-1 / 4 J}$. Thus $T_{K}$ can be tuned to the experimentally accessible regime by appropriately increasing the lead-TA conductances and making an atom with sufficiently large level spacing. A triangular atom with side $200 \mathrm{~nm}$ would be challenging but feasible to fabricate, and should satisfy the level spacing requirement.

It is interesting to study the effect of a Zeeman field, $H_{\mathrm{TA}} \rightarrow H_{\mathrm{TA}}-B S^{z}$. Experimentally, this corresponds to a field applied parallel to the surface of a lateral artificial atom [17]. For $B>T_{K}$ spin fluctuations on the atom are suppressed. Orbital fluctuations remain allowed, and in the spin channel parallel to $B$ a purely orbital Kondo state is formed. For $J_{\alpha \beta}^{\mu \nu} \approx J \delta_{\beta}^{\mu} \delta_{\alpha}^{\nu}$ we find that the orbital Kondo temperature is somewhat suppressed compared to that of the $S U(4)$ Kondo state, $T_{K}(B \rightarrow \infty) \approx \Delta e^{-1 / 2 J} \sim T_{K}(B=0)^{2} / \Delta$.

In contrast to the $\mathrm{SU}(4)$ Kondo state, this or- bital state is characterized by phase shifts $\delta_{\uparrow}=$ $\pi / 2$ and $\delta_{\downarrow}=0$. Interestingly, at $T=0$ the conductance $G$ is approximately field-independent, since $\delta_{\uparrow}+\delta_{\downarrow}=\pi / 2$ as a consequence of the Friedel sum rule. Thus the conductance in this regime should increase with decreasing temperature even for $T<B$ and should saturate below $T_{K}(B \rightarrow \infty)$, a clear signature of a purely orbital Kondo state.

The polarization of the transmitted current, on the other hand, $P=\sin ^{2} \delta_{\uparrow}-\sin ^{2} \delta_{\downarrow}$, does depend on $B$, and the current becomes almost fully polarized for $B>T_{K}$ at $T=0$. Therefore the triangular atom in this regime could be used as a perfectly spin filter with high conductance.

Distorting the shape of the TA, or applying a small magnetic field perpendicular to the surface, splits the $\Gamma_{3}$ multiplet. As with application of a parallel field, the SU(4) Kondo is destroyed, replaced by a standard spin- $1 / 2$ Kondo state with reduced Kondo temperature. The conductance tensor in this case becomes more complicated, however, and cannot be parametrized as before.

Stability. - Finally, let us comment on the conditions under which the SU(4) and orbital Kondo states can be observed. Clearly, it is experimentally very important to have as perfect triangular symmetry as possible. Departures from symmetry have two major consequences: (a) The couplings $J_{\alpha \beta}^{\mu \nu}$ become anisotropic. Fortunately, this has little importance, since the $\mathrm{SU}(4)$ state is stable in the renormalization group sense, i.e., these differences can be neglected in the Kondo regime. (b) The $\Gamma_{3}$ multiplet splits. This effect is insignificant until the splitting is larger than $T_{K}$, which can be a substantial fraction of the average level spacing. We therefore believe that these new Kondo states can indeed be experimentally observed in a triangular artificial atoms.

Summary. - In a perfectly triangular artificial atom many of the single particle orbital states are degenerate by symmetry. We have shown that such orbital degeneracy can result in the formation of an $S U(4)$ symmetric Kondo state with phase shifts $\delta=\pi / 4$, or a purely orbital Kondo state in the presence of a strong external parallel magnetic field. The triangular atom acts as a spin polarizer in this regime. We have also sug- 
gested using triangular atoms to study systematically the singlet-triplet transition described in Ref. [4] and apparently observed in Ref. [11].

Acknowledgments. - We are grateful to B.I. Halperin and W. Hofstetter for stimulating discussions. This research has been supported by NSF Grants Nos. DMR-9985978 and Hungarian Grants No. OTKA F030041, T038162, and N31769.

\section{REFERENCES}

1. R.C. Ashoori, Nature 379, 413 (1996).

2. D.H. Cobden and J. Nygard, Phys. Rev. Lett. 89046803 (2002).

3. M. Pustilnik and L.I. Glazman, Phys. Rev. Lett. 87, 216601/1 (2001).

4. W. G. van der Wiel, S. De Franceschi, J. M. Elzerman, S. Tarucha, L. P. Kouwenhoven, J. Motohisa, F. Nakajima, and T. Fukui, Phys. Rev. Lett. 88, 126803 (2002).

5. W. Hofstetter and H. Schoeller, Phys. Rev. Lett. 88, 016803/1 (2002).

6. Y. Alhassid, Rev. Mod. Phys. 72, 895 (2000).

7. A. M. Chang, H. U. Baranger, L. N. Pfeiffer, K. W. West, and T. Y. Chang, Phys. Rev. Lett. 76, 1695 (1996); J. A. Folk, S. R. Patel, S. F. Godijn, A. G. Huibers, S. M. Cronenwett, C. M. Marcus, K. Campman, and A. C. Gossard, Phys. Rev. Lett. 76, 1699 (1996); S. R. Patel, S. M. Cronenwett, D. R. Stewart, A. G. Huibers, C. M. Marcus, C. I. Duruoz, J. S. Harris, K. Campman, and A. C. Gossard, Phys. Rev. Lett. 80, 4522 (1998).

8. S. Tarucha, D.G. Austing, T. Honda T, R.J. van der Hage, and L.P. Kouwenhoven, Phys. Rev. Lett. 773613 (1996).

9. L. Borda, G. Zaránd, W. Hofstetter, B.I. Halperin, and Jan von Delft, condmat/0207001 (to appear in Phys. Rev. Lett.); A similar system has been inversigated more recently in K. Le Hur and P. Simon, condmat/0210342.

10. A. Yacoby et al., Phys. Rev. Lett. 74, 4047 (1995).

11. A. Kogan, G. Granger, M.A. Kastner, D. Goldhaber-Gordon, and Hadas Shtrikman, cond-mat/0208268 (2002).
12. V.D. Averin, A.N. Korotkov, and K.K. Likharev, Phys. Rev. B 44, 6199 (1991); C.W.J. Beenakker, Phys. Rev. B 44, 1646 (1991).

13. D. Goldhaber-Gordon, et al., Nature 391, 156 (1998); S.M. Cronenwett, T.H. Oosterkamp, and L.P. Kouwenhoven, Science 281, 540 (1998).

14. B. Coqblin and J. R. Schrieffer, Phys. Rev. 185, 847 (1969).

15. P. Nozieres and A. Blandin, J. Physique 41, 193 (1980).

16. R. Landauer, IBM J. Res. Dev. 1, 223 (1957); D.S. Fisher and P. Lee, Phys. Rev. Lett. 23, 6851 (1981).

17. A small magnetic field perpendicular to the surface would split the orbital degeneracy $\Gamma_{3}$ multiplet too. 